\title{
Preparation of Nitrosulfonated Humic Acid-based Ion-imprinted Polymer and Study on Its Adsorption Properties
}

\section{An Wang}

North China University of Science and Technology

Xiaoran Sun ( $\nabla$ xiaoranhb@sohu.com )

North China University of Science and Technology

\section{Yinuo Yan}

North China University of Science and Technology

\section{Simeng Bian}

North China University of Science and Technology

\section{Kaili Fan}

North China University of Science and Technology

\section{Zheng Zhao}

North China University of Science and Technology

Lihua Han

North China University of Science and Technology

Hongzhou Shang

North China University of Science and Technology

\section{Research Article}

Keywords: humic acid, imprinted, kinetics, thermodynamics

Posted Date: June 10th, 2021

DOI: https://doi.org/10.21203/rs.3.rs-589502/v1

License: (1) (1) This work is licensed under a Creative Commons Attribution 4.0 International License. Read Full License 


\title{
Preparation of nitrosulfonated humic acid-based ion-imprinted polymer and
}

\section{study on its adsorption properties}

\author{
An Wang ${ }^{\mathrm{a}}$, Yinuo Yan ${ }^{\mathrm{a}}$, Xiaoran Sun ${ }^{\mathrm{a}, *}$, Simeng Bian ${ }^{\mathrm{a}, *}$, Kaili Fan ${ }^{\mathrm{a}}$, Zheng Zhao ${ }^{\mathrm{a}}$, Lihua Han ${ }^{\mathrm{a}}$, Hongzhou Shang \\ ${ }^{a}$ School of Chemical Engineering, North China University of Science and Technology, Tangshan 063210, China \\ ${ }^{\mathrm{b}}$ School of material science and Engineering, North China University of Science and Technology, Tangshan 063210, China \\ * Corresponding author at: School of Chemical Engineering, North China University of Science and Technology, Tangshan \\ 063210, China
}

E-mail addresses: xiaoranhb@souhu.com; bsm0304@163.com

Abstract: Ion imprinted polymer, a kind of high molecular organic polymer, which can separate and enrich various metal ions in water by changing the adsorption conditions. Used humic acid as raw materials, used $\mathrm{HNO}_{3}$ and $\mathrm{H}_{2} \mathrm{SO}_{4}$ as modifiers to synthesize nitrosulfonated humic acid, then used it as substrate, NIPAM as the functional monomer, $\mathrm{Pb}^{2+}$ as the template ion, MBA as the crosslinking agent, $\mathrm{K}_{2} \mathrm{~S}_{2} \mathrm{O}_{8}$ and $\mathrm{NaHSO}_{3}$ as initiators to prepare lead ion imprinted polymers after cross-linking reaction. Used SEM, FT-IR, TG-DSC to characterize the obtained product, and explored the structure and performance of the product. The adsorption performance of IIPs (NSA/NIPAM) was studied by ultraviolet-visible spectrophotometry (UV-vis). The results show that the maximum adsorption capacity of IIPs (NSA/NIPAM) for $\mathrm{Pb}^{2+}$ is $81.33 \mathrm{mg} / \mathrm{g}$, the optimal number of cycles of IIPs (NSA/NIPAM) is 10 , In the presence of competing ions $\mathrm{Cu}^{2+}$ and $\mathrm{Cd}^{2+}$, selectivity coefficients are $18.04\left(\mathrm{~Pb}^{2+} / \mathrm{Cu}^{2+}\right)$ and $19.39\left(\mathrm{~Pb}^{2+} / \mathrm{Cd}^{2+}\right)$. The results of simulating the thermodynamic adsorption process of the polymers with Langmuir and Freundlich thermodynamic adsorption models show that the imprinted polymer conform to the second-order kinetic model and the Langmuir thermodynamic model, and belong to monolayer chemisorption.

Keywords: humic acid, imprinted, kinetics, thermodynamics

\section{Introduction}

Ion imprinting technology refers to a technology that uses different metal ions as template ions, combines them with functional monomers through electrostatic interaction, chemical adsorption, and complex chelation, then removes the template ions through a special method, and the corresponding holes generated by it will have a specific recognition function for metal ions ${ }^{[1-3]}$. The ion imprinted polymer synthesized by this technology has broad application prospects in the detection, separation and enrichment of heavy metals ${ }^{[4-6]}$.

The general method for preparing ion imprinted polymers is to first pre-polymerize template ions and functional monomers in a certain medium, then perform cross-linking polymerization, and finally elute template ions to obtain imprinted polymers. However, the template ions of the ion imprinted polymer prepared by this method are not easy to elute, and the recovery is difficult ${ }^{[7,8]}$. Grafting the imprinted polymer on a solid surface (matrix) has become a new method for preparing ion imprinted polymers, which can prepare ion imprinted polymer with easily eluting template ion, large specific surface area, high template ion recognition ability, and good thermal stability ${ }^{[9]}$. Currently, the main substrates used in the preparation of ion-imprinted polymers are carbon nanotubes ${ }^{[10-12]}$, silica ge ${ }^{[13-15]}, \mathrm{Fe}_{3} \mathrm{O}_{4}{ }^{[16-20]}$, and montmorillonite ${ }^{[21-24]}$.

Humic acid is an environmentally friendly natural polymer with a complex structure and a variety of active functional groups, which is widely found in soil, swamps, silt, and weathered coal. The molecular structure of humic acid contains various functional groups, which has good chelating, complexing and ion exchange abilities, and is easy to enrich and separate heavy metal ions ${ }^{[25]}$. Various adsorbents synthesized with humic acid or modified humic acid as raw materials have strong heavy metal ion adsorption performance, realize the enrichment and separation of heavy metal ions, and alleviate heavy metal ion pollution ${ }^{[26]}$.

Using humic acid as raw material, a new type of ion imprinting material with good enrichment and separation ability for trace and ultra-trace heavy metal ions is synthesized, which can achieve the adsorption 
and separation of harmful heavy metal ions in the environment and enrich precious metals. By studying the adsorption performance of ion imprinted polymers, an adsorption model is established to explain the specific adsorption performance and adsorption mechanism of polymers for $\mathrm{Pb}^{2+}$, provide basic parameters for industrial enrichment of trace and ultra-trace heavy metal ions and new methods for environmental pollution.

\section{Material and methods}

\subsection{Reagents}

Humic acid (HA, molecular formula: $\mathrm{C}_{9} \mathrm{H}_{9} \mathrm{NO}_{6}$, Shandong West Asia Chemical Industry Co., Ltd.); Concentrated nitric acid $\left(\mathrm{HNO}_{3}, \mathrm{w}=65-68 \%\right.$, Beijing Reagent Beijing Chemical Plant); Concentrated sulfuric acid $\left(\mathrm{H}_{2} \mathrm{SO}_{4}, \mathrm{w}=95-98 \%\right.$, Tianjin Kaixin Chemical Industry Co., Ltd.) N-isopropylacrylamide, N,Nmethylenebisacrylamide, lead nitrate, 4-(2-Pyridylazo)-resorcinol mono sodium salt (PAR), AR, Shanghai Aladdin Biochemical Technology Co., Ltd.; Potassium persulfate, sodium bisulfite, sodium hydroxide, absolute ethanol, concentrated hydrochloric acid, disodium ethylenediaminetetraacetic acid (EDTA), AR, Shanghai Macleans reagent.

\subsection{Preparation of nitrosulfonated humic acid-based ion-imprinted polymer}

Accurately weigh $5 \mathrm{~g}$ of humic acid (HA) into a three-necked flask, add $50 \mathrm{~mL}$ of $25 \%$ concentrated $\mathrm{H}_{2} \mathrm{SO}_{4}$ and $50 \mathrm{~mL}$ of $15 \%$ concentrated $\mathrm{HNO}_{3}$, under airtight protection, heat to $80{ }^{\circ} \mathrm{C}$ for condensation and reflux $2 \mathrm{~h}$. After the reaction, cool to room temperature, transfer the obtained solid-liquid mixture to the reaction kettle, put the reaction kettle in the blast drying oven, heat up to $105^{\circ} \mathrm{C}$ for $6 \mathrm{~h}$, after cooling to room temperature, centrifuge at $8000 \mathrm{r} / \mathrm{min}$ for $10 \mathrm{~min}$, separate the solid product, wash the product twice with distilled water and dry it under vacuum for $24 \mathrm{~h}$ to obtain nitrosulfonated humic acid (NSA).

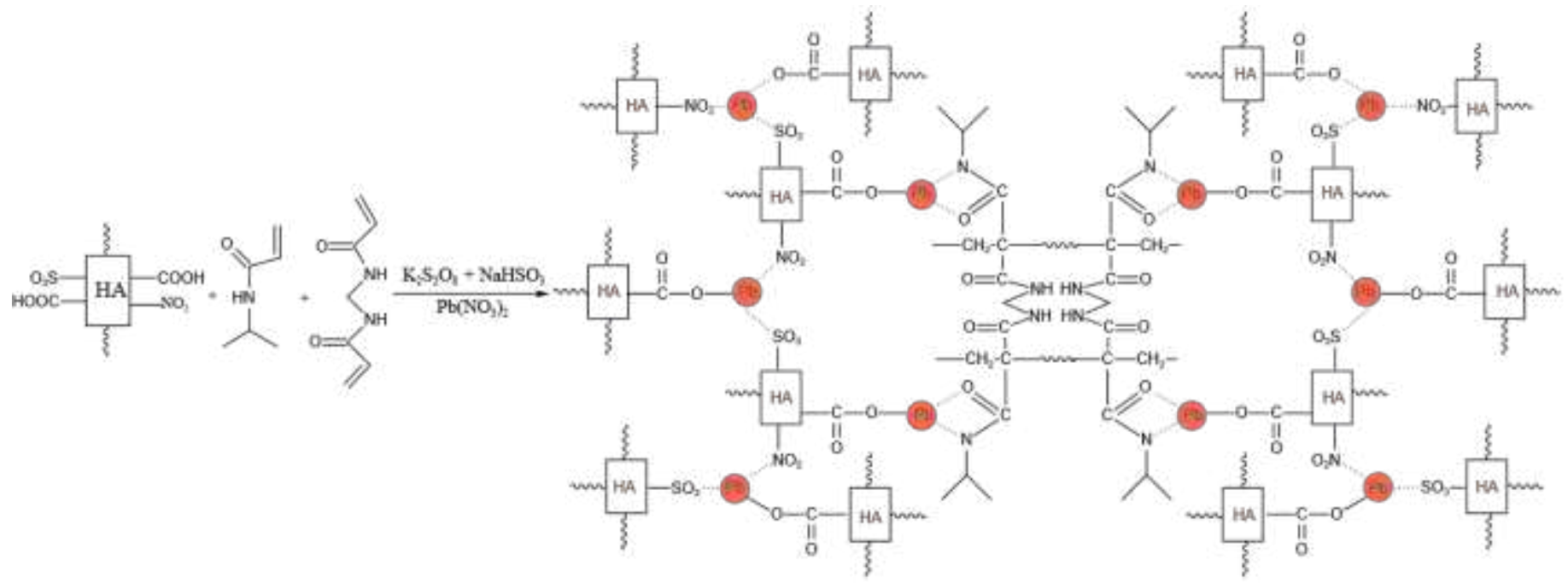

Fig.1. Preparation process of imprinted polymer.

Weigh $0.02 \mathrm{~g} \mathrm{~K}_{2} \mathrm{~S}_{2} \mathrm{O}_{8}$ and $0.01 \mathrm{~g} \mathrm{NaHSO}_{3}$ in a small beaker, add $20 \mathrm{~mL}$ of distilled water, and dissolve by ultrasound to prepare a composite initiator. Weigh $0.3 \mathrm{~g}$ of NSA and $0.15 \mathrm{~g}$ of NIPAM in a three-necked flask, add $80 \mathrm{~mL}$ of distilled water, heat to $40{ }^{\circ} \mathrm{C}$ and stir for $0.5 \mathrm{~h}$, then add $0.15 \mathrm{~g}$ of $\mathrm{Pb}\left(\mathrm{NO}_{3}\right)_{2}$ and $0.03 \mathrm{~g}$ of cross-linking agent MBA, continue to mix and stir for $0.5 \mathrm{~h}$, slowly drop the composite initiator into the threenecked flask with a dropping funnel, and pass nitrogen protection. After the reaction, cool to room temperature and filter under reduced pressure to obtain a solid product, wash it with distilled water 2 to 3 times. The product was soaked in the mixed solution of $2 \%$ hydrochloric acid solution and $0.5 \mathrm{~mol} / \mathrm{L}$ EDTA for $24 \mathrm{~h}$, filtered under reduced pressure to obtain a solid, and dried under vacuum to obtain lead ion imprinted polymer 
IIPs (NSA/NIPAM). The preparation method of the corresponding non-ionic imprinted polymer NIIPs (NSA/NIPAM) is the same as the above steps, and the template lead ion is not added during the reaction. The preparation process of imprinted polymer is shown in Fig. 1.

\subsection{Adsorption experiment}

The adsorption performance of imprinting material IIPs (NSA/NIPAM) on $\mathrm{Pb}^{2+}$ was measured by the water bath shaking method. Add a certain concentration of IIPs (NSA/NIPAM) to a shaking bottle containing a certain concentration of $\mathrm{Pb}^{2+}$ solution, filter the solution after a period of time, measure the $\mathrm{Pb}^{2+}$ concentration in the solution, and calculate the adsorption amount and removal rate of the material. The calculation formulas are as follows:

$$
\begin{aligned}
& q_{e}=\frac{\left(c_{0}-c_{e}\right) V}{m} \\
& E=\frac{c_{0}-c_{e}}{c_{0}} \times 100 \%
\end{aligned}
$$

Where $\mathrm{q}_{\mathrm{e}}$ is the equilibrium adsorption capacity, $\mathrm{mg} / \mathrm{g} ; \mathrm{c}_{0}$ is the initial concentration of $\mathrm{Pb}^{2+}$ ions in the solution, $\mathrm{mg} / \mathrm{L}$; $\mathrm{c}_{\mathrm{e}}$ is the equilibrium concentration of $\mathrm{Pb}^{2+}$ ions in the solution, $\mathrm{mg} / \mathrm{L} ; \mathrm{V}$ is the volume of the solution, $\mathrm{L}$; $\mathrm{E}$ is the removal rate of $\mathrm{Pb}^{2+}, \% ; \mathrm{m}$ is the mass of imprinted material or non-imprinted material, g.

\subsection{Structure and characterization}

Infrared spectroscopy (FT-IR) (VERTEX70, Bruker, Germany), using potassium bromide tablet method, the HA, NSA, IIPs (NSA/NIPAM) were characterized by infrared spectrometer, the test range is $400-4000 \mathrm{~cm}^{-}$ 1; Scanning electron microscopy (SEM) (S-4800, Hitachi, Japan), HA and IIPs (NSA/NIPAM) are sprayed with gold, and the morphology and structure of the sample surface are observed by scanning electron microscope; Thermogravimetric analysis (TG-DSC) (STA449F5, Netzsch), under the protection of nitrogen atmosphere, the temperature range is $20-800{ }^{\circ} \mathrm{C}$, the heating rate is $10{ }^{\circ} \mathrm{C} / \mathrm{min}$, thermogravimetric analysis of HA and IIPs (NSA/NIPAM) using thermogravimetric analyzer; Ultraviolet-visible spectrophotometer (UVvis) (Uv-1900i, Tsushima Instruments Suzhou Co., Ltd.).

\section{Results and discussion}

\subsection{Infrared Spectroscopy (FT-IR)}

Through infrared spectroscopy, HA, NSA, IIPs (NSA/NIPAM) were analyzed by infrared, and the results are shown in Fig. 2. Among them, $2990 \mathrm{~cm}^{-1}$ and $2910 \mathrm{~cm}^{-1}$ are the stretching vibration peaks of $-\mathrm{CH}_{3}$ and $\mathrm{CH}_{2}$ - respectively. The characteristic peak of sulfonic acid group at $1060 \mathrm{~cm}^{-1}$ indicates that the modified sulfonic acid group of humic acid by concentrated sulfuric acid was successfully grafted onto humic acid. $1060 \mathrm{~cm}^{-1}$ and $1690 \mathrm{~cm}^{-1}$ are the stretching vibration peaks of the nitro group, which may be due to the blue shift of the characteristic peak caused by the amide bond during the reaction. The peak shape at $875 \mathrm{~cm}^{-1}$ on IIPs (NSA/NIPAM) is not sharp enough and the peak with medium intensity is the stretching vibration peak of - $\mathrm{CN}$ - on $\mathrm{N}$-isopropylacrylamide, which indicates that $\mathrm{N}$-isopropylacrylamide successfully polymerized with nitrosulfonated humic acid, and successfully synthesized ion imprinted polymer. 


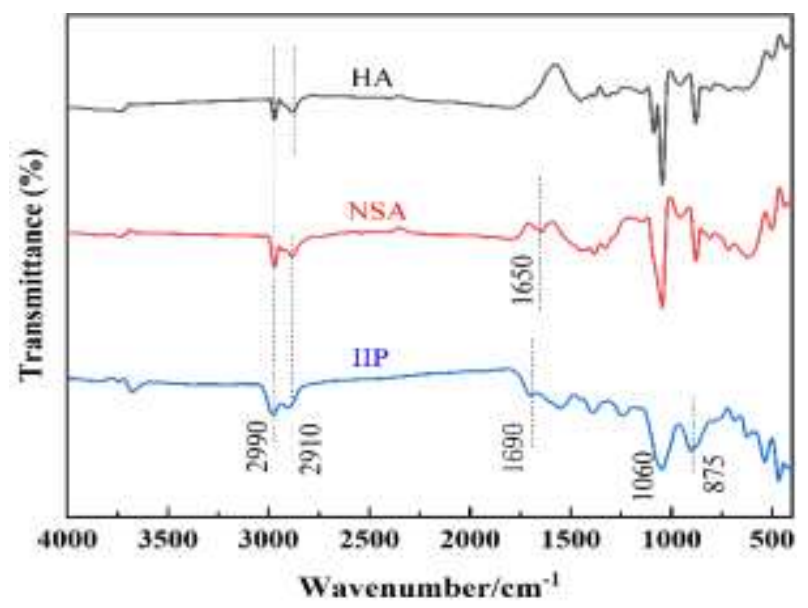

Fig. 2. Infrared spectra of HA, NSA, IIPs (NSA/NIPAM).

\subsection{Scanning electron microscope (SEM)}

The structure of NSA and IIPs (NSA/NIPAM) were characterized by scanning electron microscopy. Fig. $3(a, b)$ are the scanning electron micrographs of NSA at 5000 and 10000 times respectively. Observing the morphological characteristics of the sample, it is found that the surface of SA is relatively smooth, the outline is clear, and it presents a state of irregular block accumulation. Fig. 3 (c, d) are the scanning electron micrographs of IIPs (NSA/NIPAM) at 5000 and 10000 times respectively. Through comparison, it can be intuitively found that the surface of IIPs (NSA/NIPAM) has become rough, and it can be seen that there is a similar effect of polymer agglomeration. Under high-power observation, it is found that there are some holes on the surface of the polymer, which may be the specific recognition sites left by the imprinted polymer after the template ion is removed, indicating that the imprinted polymer was successfully made.

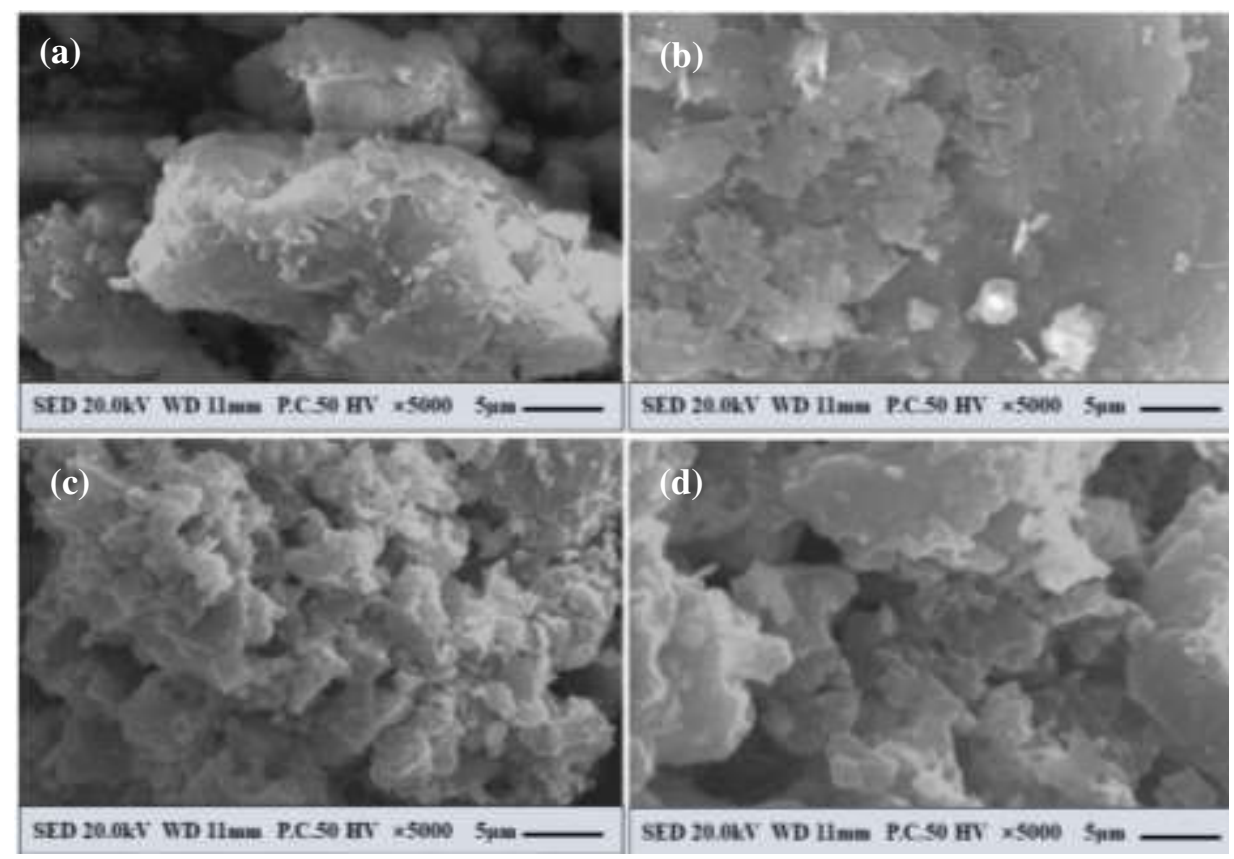

Fig. 3. NSA $\times 5000$ (a); NSA $\times 10000$ (b); IIPs(NSA/NIPAM) $\times 5000$ (c) and $\mathrm{Pb}^{2+}-\operatorname{IIPs}(\mathrm{NSA} / \mathrm{NIPAM}) \times 10000$ (d) Scanning electron micrograph

\subsection{Thermogravimetric analysis (TG-DSC)}

Fig. $4(a, b)$ is the thermogravimetric analysis diagram of NSA, IIPs (NSA/NIPAM). It can be seen from the figure that NSA had a rapid weight loss rate at $100{ }^{\circ} \mathrm{C}$, because the sample may contain a small amount of water molecules, when the temperature reaches $100{ }^{\circ} \mathrm{C}$, the volatilization of water molecules causes weight 
loss. NSA loses about $20 \%$ of its weight between $300-500{ }^{\circ} \mathrm{C}$, which may be caused by thermal decomposition of the carboxyl, hydroxyl, and sulfonic acid groups on the sulfonated humic acid; In contrast, IIPs (NSA/NIPAM) has only a small weight loss rate within $100{ }^{\circ} \mathrm{C}$, this is because only a small amount of water in the sample evaporates, and the sample loses about $5 \%$ between 300 and $500{ }^{\circ} \mathrm{C}$. IIPs (NSA/NIPAM) has a large weight loss rate at $600{ }^{\circ} \mathrm{C}$, the weight loss is about $15 \%$, which may be caused by the thermal instability of IIPs (NSA/NIPAM), a small amount of organic matter remaining in the polymer, which is easily decomposed at $600{ }^{\circ} \mathrm{C}$. It can be seen that the thermal stability of IIPs (NSA/NIPAM) is not good, and the organic content on the surface of the imprinted polymer is about $25 \%$.
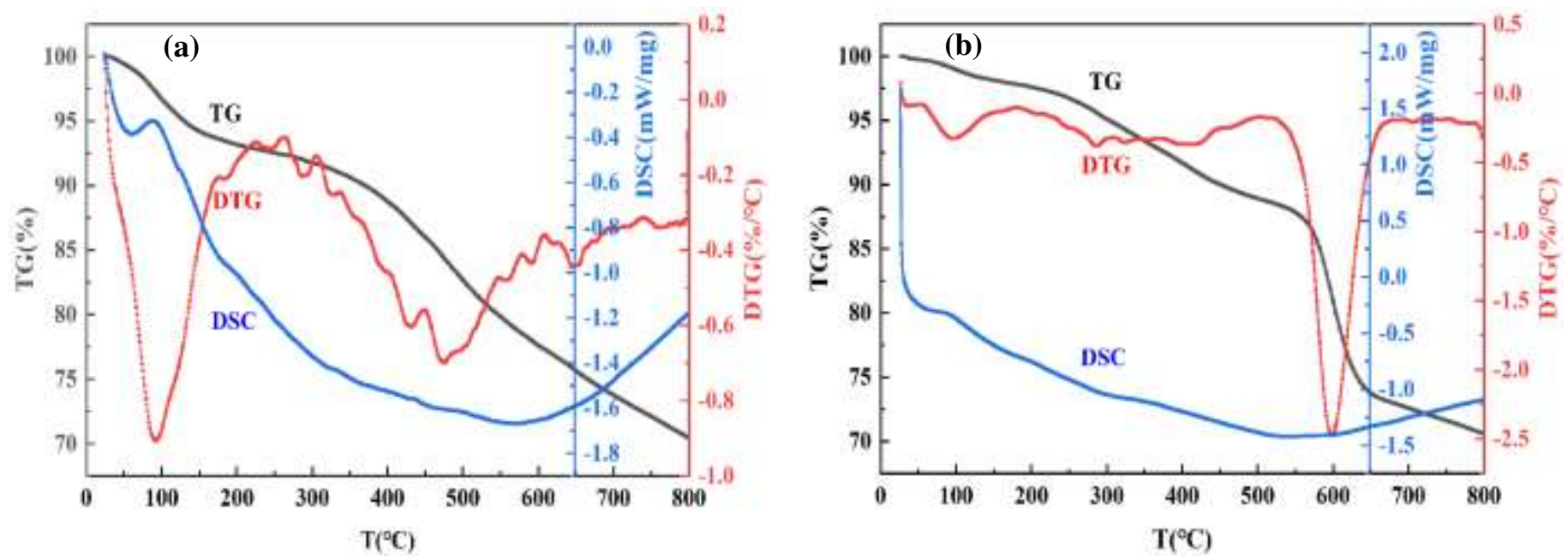

Fig.4. NSA (a) and IIPs(NSA/NIPAM ) (b) TG-DTG-DSC thermogravimetric analysis chart

\subsection{Analysis of adsorption performance of IIPs (NSA/NIPAM)}

Fig. 5(a) shows the effect of the amount of different IIPs (NSA/NIPAM) on the adsorption capacity. It can be seen from the figure that with the increase in the amount of IIPs (NSA/NIPAM), the adsorption capacity first increases and then decreases. This is because in the initial stage, the amount of IIPs (NSA/NIPAM) is small, and it is not enough to absorb all lead ions in the solution; as the amount of IIPs (NSA/NIPAM) increases, the amount of adsorption also presents a proportional relationship, when the amount of IIPs (NSA/NIPAM) is $10 \mathrm{mg}$, the adsorption capacity reaches the maximum $81.33 \mathrm{mg} / \mathrm{g}$; then with the increase of imprinted polymer, the adsorption capacity does not increase but decreases, because in the adsorption system the adsorption equilibrium of IIPs (NSA/NIPAM) has been reached, no matter how the IIPs (NSA/NIPAM) increase, the content of lead ions in the solution is fixed, therefore, continuing to add IIPs (NSA/NIPAM) will cause the amount of lead ions adsorbed per unit of adsorbent to decrease.

Fig. 5(b) shows the effect of different temperatures on the adsorption capacity. It can be seen from the figure that as the temperature increases, the adsorption capacity of IIPs (NSA/NIPAM) on lead ions shows a trend of first increasing and then decreasing. This is because at a lower temperature of $10{ }^{\circ} \mathrm{C}$, the speed of molecular movement is low, the probability of contact between IIPs (NSA/NIPAM) and lead ions in the solution is low, and the reaction rate is slow, resulting in a low adsorption capacity; as the temperature rises, the speed of molecular movement speeds up in the solution system and the reaction rate becomes faster, so the adsorption capacity also increases, and finally the adsorption capacity reaches a maximum of $81.25 \mathrm{mg} / \mathrm{g}$ at $30{ }^{\circ} \mathrm{C}$; then as the temperature continues to rise, the adsorption capacity decreases instead, the reason is that the higher the temperature, the faster the movement rate of the reaction molecules and the stronger the reaction activity, which causes the lead ions that have just been adsorbed to desorb quickly, so the adsorption capacity is getting lower and lower.

Fig. 5(c) shows the effect of $\mathrm{pH}$ on the adsorption capacity. With the increase of $\mathrm{pH}$, the adsorption capacity of IIPs (NSA/NIPAM) shows a trend of first increasing and then decreasing. This may be because too much $\mathrm{H}+$ or $\mathrm{OH}^{-}$exists in the solution system, which will compete with IIPs (NSA/NIPAM) for $\mathrm{Pb}^{2+}$ and 
weaken the adsorption capacity of imprinted polymers, but a small amount of $\mathrm{H}^{+}$may promote the adsorption of $\mathrm{Pb}^{2+}$ by IIPs (NSA/NIPAM). Therefore, under the weakly acidic condition of $\mathrm{pH}=6$, the adsorption capacity of IIPs (NSA/NIPAM) reaches the maximum of $81.14 \mathrm{mg} / \mathrm{g} ; \mathrm{OH}^{-}$will also have a certain influence on the adsorption capacity of the solution, this may be because a small amount of $\mathrm{OH}^{-}$will attract the $\mathrm{Pb}^{2+}$ charge, resulting in incomplete adsorption of IIPs (NSA/NIPAM), so with the increase of $\mathrm{pH}$ in the adsorption capacity will also slightly decrease.

Fig. 5(d) shows the cyclic adsorption capacity of IIPs (NSA/NIPAM) for $\mathrm{Pb}^{2+}$. From the figure, it can be found that the adsorption capacity of IIPs (NSA/NIPAM) for $\mathrm{Pb}^{2+}$ is $81.33-76.11 \mathrm{mg} / \mathrm{g}$. The adsorption capacity decreases slightly as the number of cycles increases, this may be due to the loss of IIPs (NSA/NIPAM) after many cycles, so the adsorption capacity decreased slightly, but basically remained at a certain level, indicating that IIPs (NSA/NIPAM) have good recycling capacity.
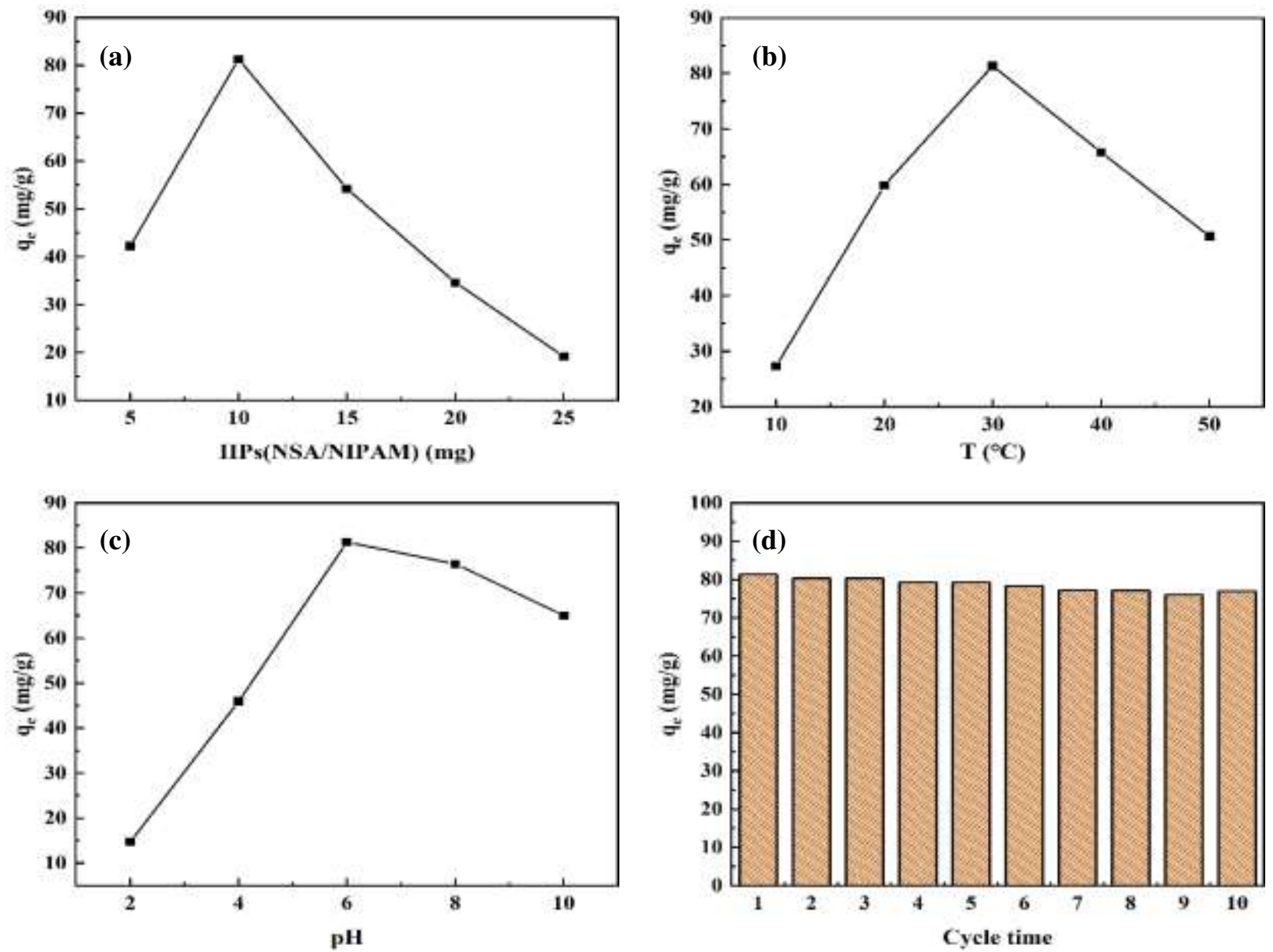

Fig.5. The effect of the amount of IIPs (NSA/NIPAM) on the adsorption capacity (a); The effect of temperature on adsorption capacity (b); The effect of $\mathrm{pH}$ on adsorption capacity (c); Cyclic adsorption capacity (d)

\subsection{Selective adsorption analysis}

Weigh $10 \mathrm{mg}$ of IIPs (NSA/NIPAM) and NIIPs (NSA/NIPAM), put them into $100 \mathrm{~mL}$ of $10 \mathrm{mg} / \mathrm{L}$ mixed $\mathrm{Pb}^{2+}, \mathrm{Cu}^{2+}, \mathrm{Cd}^{2+}$ solution for 100 minutes, shake, and then stand and filter for detection. The content of the three metal ions in the solution, the calculation formula for calculating the adsorption capacity, static distribution coefficient $\left(\mathrm{K}_{\mathrm{d}}\right)$, and selectivity coefficient $(\mathrm{K})$ is listed below:

$$
\begin{aligned}
& K_{d}=\frac{q_{e}}{c_{e}} \\
& K=\frac{K_{d}\left(M^{n+}\right)}{K_{d}\left(N^{n+}\right)}
\end{aligned}
$$


Where $\mathrm{K}_{\mathrm{d}}$ is static distribution coefficient; $\mathrm{K}$ is selectivity coefficient; $K_{d}\left(M^{n+}\right)$ is partition coefficient of template ion; $K_{d}\left(N^{n+}\right)$ is partition coefficient of competing ions.

Table 1 Selective adsorption of IIPs(NSA/NIPAM) and NIIPs(NSA/NIPAM)

\begin{tabular}{cccc}
\hline \multirow{2}{*}{ Metal ion } & \multicolumn{3}{c}{ IIPs(NSA/NIPAM)/NIIPs(NSA/NIPAM) } \\
\cline { 2 - 4 } & $\mathrm{q}_{\mathrm{e}}(\mathrm{mg} / \mathrm{g})$ & $\mathrm{K}_{\mathrm{d}}$ & $\mathrm{K}$ \\
\hline $\mathrm{Pb}^{2+}$ & $78.30 / 14.35$ & $3.4493 / 0.1675$ & ------- \\
$\mathrm{Cu}^{2+}$ & $16.05 / 14.88$ & $0.1912 / 0.1748$ & $18.04 / 0.9582$ \\
$\mathrm{Cd}^{2+}$ & $15.10 / 14.45$ & $0.1779 / 0.1689$ & $19.39 / 0.9917$ \\
\hline
\end{tabular}

It can be seen from Table 1 that the selective adsorption coefficients of IIPs (NSA/NIPAM) are 18.04 $\left(\mathrm{Pb}^{2+} / \mathrm{Cu}^{2+}\right)$ and $19.39\left(\mathrm{~Pb}^{2+} / \mathrm{Cd}^{2+}\right)$, both of which are far greater than 1 . The selective adsorption coefficients of NIIPs (NSA/NIPAM) are $0.9582\left(\mathrm{~Pb}^{2+} / \mathrm{Cu}^{2+}\right)$ and $0.9917\left(\mathrm{~Pb}^{2+} / \mathrm{Cd}^{2+}\right)$, both of which are less than 1, which indicates that the selective adsorption capacity of IIPs (NSA/NIPAM) for $\mathrm{Pb}^{2+}$ is higher than that of NIIPs (NSA/NIPAM).

\subsection{Adsorption kinetic}

Weigh $10 \mathrm{mg}$ of IIPs (NSA/NIPAM) into $100 \mathrm{~mL}$ of $10 \mathrm{mg} / \mathrm{L} \mathrm{Pb}\left(\mathrm{NO}_{3}\right)_{2}$ solution, keep the temperature constant, shake the solution, and take $5 \mathrm{~mL}$ of supernatant every 10 minutes to detect $\mathrm{Pb}\left(\mathrm{NO}_{3}\right)_{2}$ content to establish the adsorption performance curve. Analyze the data using first-order kinetics and second-order kinetic models. The adsorption kinetic equation are as follows:

Quasi-first order adsorption rate kinetic equation:

$$
\ln \left(q_{e}-q_{t}\right)=\ln q_{\mathrm{m}}-K_{1} t
$$

Quasi-second order adsorption rate kinetic equation:

$$
\frac{\mathrm{t}}{q_{t}}=\frac{1}{K_{2} q_{\mathrm{m}}^{2}}+\frac{t}{q_{\mathrm{m}}}
$$

Where $\mathrm{q}_{\mathrm{e}}$ is adsorption balance, $\mathrm{mg} / \mathrm{g} ; \mathrm{q}_{\mathrm{m}}$ is theoretical saturation adsorption balance, $\mathrm{mg} / \mathrm{g}$; $\mathrm{q}_{\mathrm{t}}$ is adsorption at time $\mathrm{t}, \mathrm{mg} / \mathrm{g}$; $\mathrm{t}$ is adsorption time, $\mathrm{min} ; \mathrm{K}_{1}$ is the equilibrium rate constants of quasi-first-order, $\left(\mathrm{min}^{-1}\right) ; \mathrm{K}_{2}$ is the equilibrium rate constants of quasi-second-order, $\mathrm{mg} /\left(\mathrm{g} \cdot \mathrm{min}^{1 / 2}\right)$.

The results are shown in Fig.6 between 0-120 minutes, as time increases, the adsorption capacity of IIPs (NSA/NIPAM) gradually increases to equilibrium. Before $40 \mathrm{~min}$, the amount of $\mathrm{Pb}^{2+}$ adsorbed by the polymer increased greatly, after $40 \mathrm{~min}$, the increasing trend of the amount of $\mathrm{Pb}^{2+}$ adsorbed by the polymer slowly decreased until it reached equilibrium at $100 \mathrm{~min}$, and the adsorption amount of $\mathrm{Pb}^{2+}$ by the polymer reached the maximum value of $81.33 \mathrm{mg} / \mathrm{g}$.

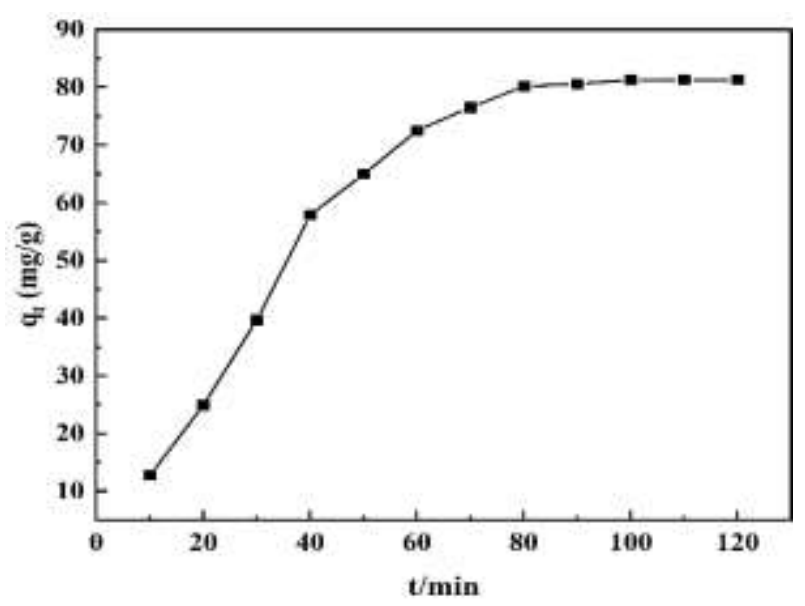

Fig. 6. Change of adsorption capacity with time

In order to further explore the adsorption mechanism of lead ions by IIPs (NSA/NIPAM), the first-order 
kinetics and second-order kinetic equations are used to fit the adsorption data. As shown in Fig. 7(a, b), the fitting results of the quasi-first-order kinetics show that before $20 \mathrm{~min}$ and after $80 \mathrm{~min}$, the deviation of the fitted data points is relatively large, $\mathrm{R}^{2}=0.9400$, the correlation is low, indicating that the adsorption of lead ions by IIPs (NSA/NIPAM) does not conform to the quasi-first-order kinetic model, so physical adsorption cannot adapt to the entire adsorption process. The quasi-second-order kinetic fitting results show that in the overall adsorption process, all data points are basically near the fitting curve, the deviation is small, and the correlation is high, $\mathrm{R}^{2}=0.9932$, and it can be determined that the adsorption of lead ions by IIPs (NSA/NIPAM) conforms to the quasi-second-order kinetic model, which indicates that it is a chemical adsorption process.
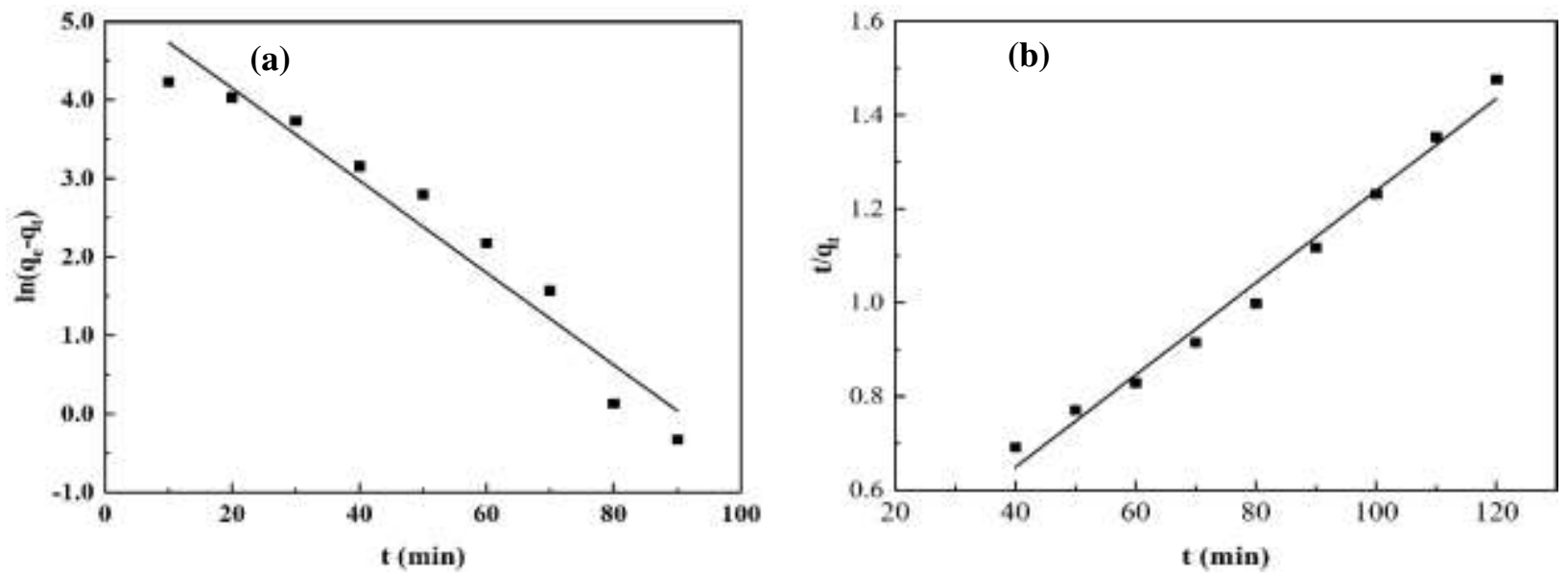

Fig. 7 Kinetics of adsorption of $\mathrm{Pb}^{2+}$ by IIPs (NSA/NIPAM) Quasi-first order kinetics fitting curve (a) and Quasi-second order kinetics fitting curve (b).

Table 2 Adsorption kinetic parameters

\begin{tabular}{|c|c|c|c|c|c|c|}
\hline \multicolumn{4}{|c|}{ Quasi-first order kinetics } & \multicolumn{3}{|c|}{ Quasi-second order kinetics } \\
\hline & $\mathrm{k}_{1} / \min ^{-1}$ & $\mathrm{R}^{2}$ & $\mathrm{q}_{\mathrm{m}} /\left(\mathrm{mg} \cdot \mathrm{g}^{-1}\right)$ & $\mathrm{k}_{2} /\left(\mathrm{g} \cdot \mathrm{mg}^{-1} \cdot \mathrm{min}^{-1}\right)$ & $\mathrm{R}^{2}$ & $\mathrm{q}_{\mathrm{m}} /\left(\mathrm{mg} \cdot \mathrm{g}^{-1}\right)$ \\
\hline $1 \pi$ & 0.0587 & 0.9400 & 204.38 & 0.00009 & 0.9932 & 101.7957 \\
\hline
\end{tabular}

\subsection{Adsorption isotherms}

Measure the lead nitrate solution of different concentrations (5, 10, 15, 20, $25 \mathrm{mg} / \mathrm{L}$ ), explore the influence of different initial concentrations on the adsorption capacity, establish the adsorption equation, and simulate the thermodynamic model.
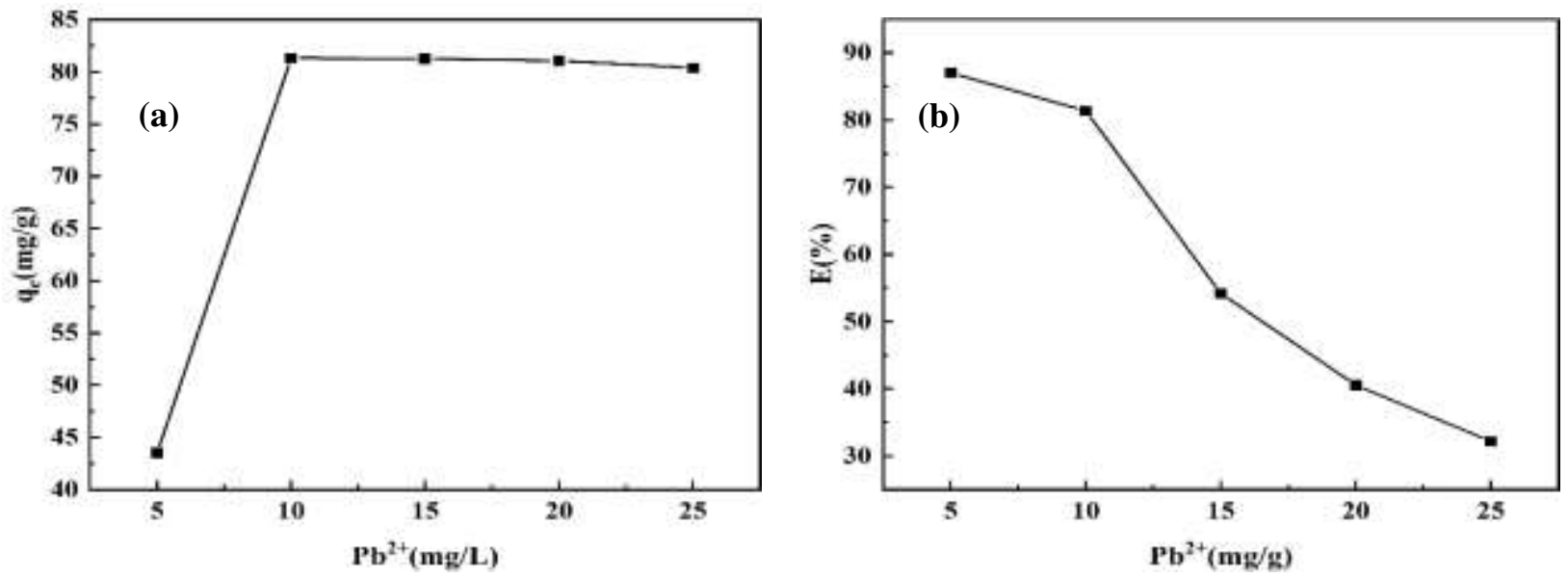

Fig. 8. The influence of the initial concentration of $\mathrm{Pb}^{2+}$ solution on the adsorption capacity (a) and removal rate (b).

Fig. $8(\mathrm{a}, \mathrm{b})$ shows the influence of the concentration of different lead ion solutions on the adsorption capacity and removal rate. It can be seen from Fig. 8(a) that with the increase of the initial concentration of the solution, the maximum adsorption capacity of IIPs (NSA/NIPAM) shows a trend that first increases and then tends to remain unchanged and slightly decreases. This is because when the initial concentration of the 
solution is $5 \mathrm{mg} / \mathrm{L}$, the dose of IIPs (NSA/NIPAM) used is too large, and the imprinted polymer is left when the lead ion adsorption equilibrium is reached, which results in a very low adsorption capacity. As the concentration increases, IIPs (NSA/NIPAM) can adsorb more lead ions, so the maximum adsorption capacity also increases, and finally reaches the maximum adsorption capacity of $81.32 \mathrm{mg} / \mathrm{g}$ when the lead ion solution concentration is $10 \mathrm{mg} / \mathrm{L}$. Then no matter how the concentration of the lead ion solution increases, the number of lead ion holes in the imprinted polymer is constant, after the IIPs (NSA/NIPAM) reaches the adsorption equilibrium, the total amount of adsorption remains unchanged, so the adsorption amount will tend to be unchanged. As the initial concentration of lead ions continues to increase, the amount of lead ions adsorbed by the imprinted polymer decreases slightly. This is because the number of lead ions near the ion imprinting point is large. The schematic diagram of the mechanism is shown in Fig. 9. Since the imprinting point can only accommodate one lead ion, too many lead ions around it will cause crowding, which will prevent the lead ions from entering the imprinting point, causing the chelating ability of the polymer to lead ions decreases, so the adsorption capacity decreases. It can be seen in Fig. 8(b) that as the lead ion concentration increases, the removal rate of lead ions gradually decreases, this is because the total amount of adsorption of IIPs (NSA/NIPAM) is constant, but the concentration of the lead ion solution is gradually increasing, so the removal rate gradually decreases.
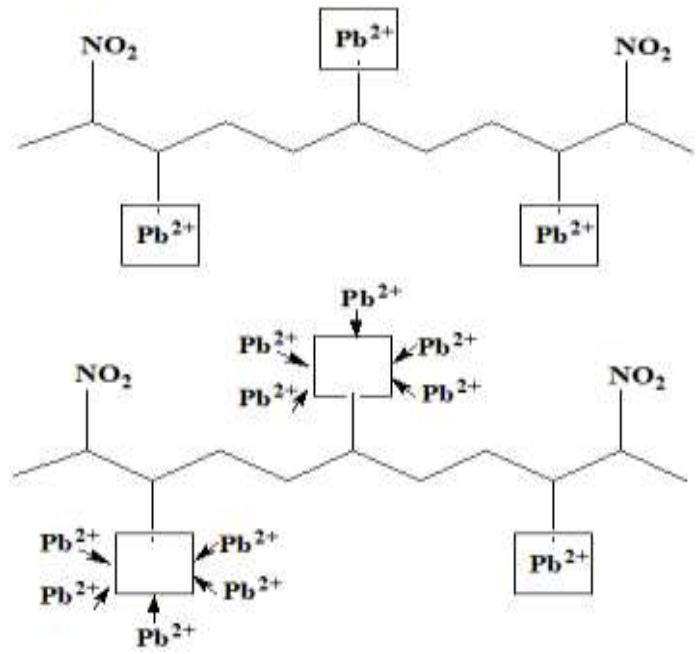

Fig. 9. Schematic diagram of adsorption mechanism.

Measure $\mathrm{Pb}\left(\mathrm{NO}_{3}\right)_{2}$ solutions with different concentrations $(5,10,15,20,25 \mathrm{mg} / \mathrm{L})$, weigh $10 \mathrm{mg}$ of IIPs (NSA/NIPAM) into $100 \mathrm{~mL}$ of $\mathrm{Pb}\left(\mathrm{NO}_{3}\right)_{2}$ with different concentrations in the solution, shake for 100 minutes and then stand and filter to detect the content of $\mathrm{Pb}\left(\mathrm{NO}_{3}\right)_{2}$ in the solution. Establish the adsorption equation, apply Langmuir and Freundlich isotherm adsorption equations to linearly fit the adsorption data, and analyze the results. The adsorption thermodynamic equation is as follows:

Langmuir isotherm adsorption model:

$$
\frac{c_{e}}{q_{e}}=\frac{1}{K_{L} q_{m}}+\frac{c_{e}}{q_{m}}
$$

Freundlich isotherm adsorption model:

$$
\ln q_{e}=\ln K_{F}+\frac{1}{n} \ln c_{e}
$$

Where $\mathrm{q}_{\mathrm{m}}$ is the theoretical saturation adsorption balance, $\mathrm{mg} / \mathrm{g} ; \mathrm{c}_{\mathrm{e}}$ is the adsorption equilibrium concentration, $\mathrm{mg} / \mathrm{L}$; $\mathrm{q}_{\mathrm{e}}$ is the adsorption balance, $\mathrm{mg} / \mathrm{g} ; \mathrm{K}_{\mathrm{L}}$ is the Langmuir equilibrium adsorption constant; $\mathrm{K}_{\mathrm{F}}$ is the Freundlich equilibrium adsorption constant; $\mathrm{n}$ is the Freundlich equation heterogeneity factor.

Fig. 10 shows the Langmuir isotherm adsorption model and Freundlich isotherm adsorption model of IIPs (NSA/NIPAM) adsorbing lead ions. It can be seen from the data in Table 3 that the Langmuir isotherm adsorption model $\left(\mathrm{R}^{2}=0.9941\right)$ has a much higher correlation than the Freundlich isotherm adsorption model $\left(\mathrm{R}^{2}=0.7384\right)$, and the simulated maximum saturated adsorption capacity is closer to the actual measured value, 
so it can be seen that the adsorption of lead ions by IIPs (NSA/NIPAM) is more in line with the Langmuir isotherm adsorption model, and the adsorption process is a monolayer adsorption.
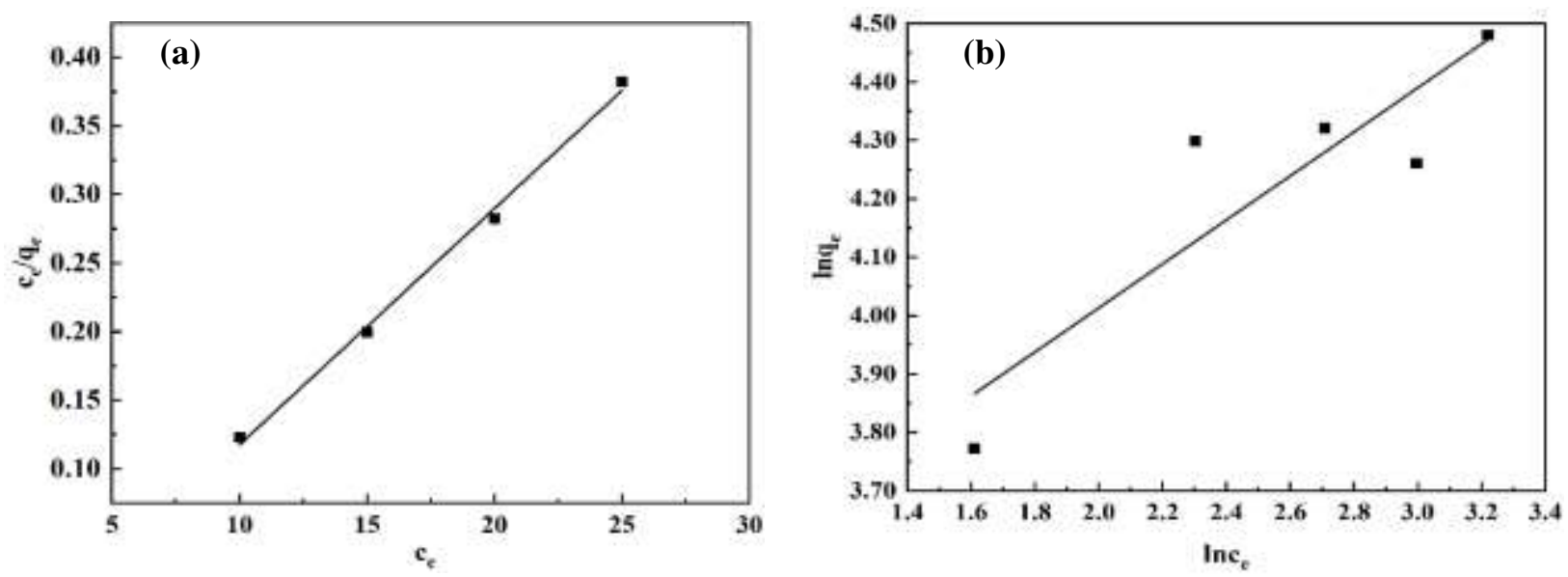

Fig. 10. The Langmuir(c) and Freundlich(d) adsorption isotherm model.

Table 3 Adsorption thermodynamic parameters

\begin{tabular}{cccccccc}
\hline & \multicolumn{3}{c}{ Langmuir adsorption isotherm model } & & \multicolumn{3}{c}{ Freundlich adsorption isotherm model } \\
\cline { 1 - 3 } IIP & $\mathrm{K}_{\mathrm{L}} /\left(\mathrm{L} \cdot \mathrm{mg}^{-1}\right)$ & $\mathrm{R}^{2}$ & $\mathrm{q}_{\mathrm{m}} /\left(\mathrm{mg} \cdot \mathrm{g}^{-1}\right)$ & & $\mathrm{K}_{\mathrm{F}} /\left(\mathrm{L} \cdot \mathrm{mg}^{-1}\right)$ & $\mathrm{R}^{2}$ & $\mathrm{n}$ \\
& 0.0546 & 0.9941 & 58.0845 & & 26.0209 & 0.7384 & 2.6523 \\
\hline
\end{tabular}

\section{Conclusions}

Through SEM, a large amount of polymer agglomerated on the surface of ion imprinted polymer IIPs (NSA/NIPAM) can be visually observed, and a small amount of pore structure can be seen on the surface of the polymer under high magnification observation, it can be inferred that the ion imprinted polymer has been synthesized; the FT-IR pattern confirms that the special functional groups of the two monomers are grafted on the surface of the imprinted polymer IIPs (NSA/NIPAM); the TG-DSC curve characterizes the thermal stability of the polymer, and it also can be found that the organic component on the surface of the imprinted polymer accounts for about $25 \%$, which has good thermal stability.

Through the exploration of the best adsorption conditions for IIPs (NSA/NIPAM) to adsorb $\mathrm{Pb}^{2+}$, the best adsorption conditions are: IIPs (NSA/NIPAM) $10 \mathrm{mg}, 100 \mathrm{~mL} 10 \mathrm{mg} / \mathrm{L}$ lead nitrate solution, $\mathrm{T}=30^{\circ} \mathrm{C}, \mathrm{pH}=6$, $\mathrm{t}=100 \mathrm{~min}$. After 10 cycles of use of IIPs (NSA/NIPAM), the adsorption capacity remains basically stable and has a good cycle adsorption capacity; when the competing ions $\mathrm{Cu}^{2+}$ and $\mathrm{Cd}^{2+}$ coexist, the selectivity coefficients are $18.04\left(\mathrm{~Pb}^{2+} / \mathrm{Cu}^{2+}\right)$ and $19.39\left(\mathrm{~Pb}^{2+} / \mathrm{Cd}^{2+}\right)$, which are far greater than 1 , indicating that IIPs (NSA/NIPAM) have good specific recognition ability. Through the data analysis of IIPs (NSA/NIPAM) adsorption of $\mathrm{Pb}^{2+}$, the adsorption model is established, and it is found that the adsorption process is more in line with the quasi-second-order kinetic model and the Langmuir isotherm adsorption model, and the adsorption process is a monolayer adsorption.

\section{Acknowledgements}

This study was funded by the key R\&D project of Hebei Province, China, "Caofeidian Port Dredged Land Resource Utilization Key Technology Integration and Demonstration". [project number 20373805D, 202006202112].

\section{Compliance with ethical standards}

Conflict of interest the authors declare that they have no conflict of interest. 


\section{Reference}

[1] Monireh B, Adil D. Highly sensitive detection of Cd (II) ions using ion-imprinted surface plasmon resonance sensors[J]. Microchemical Journal, 2020, 159:105572.

[2] Wang Z, Zhou C, Wu S, et al. Ion-Imprinted Polymer Modified with Carbon Quantum Dots as a Highly Sensitive Copper(II) Ion Probe.[J]. Polymers,2021,13(9).

[3] Ebrahim A, Hassan H, Zeinab V, et al. Synthesis, characterization and analytical applications of Ni(II) ion-imprinted polymer prepared by N-(2-hydroxyphenyl)acrylamide[J]. Journal of Polymer Research,2021,28(5).

[4] Zhang L, Xue J, Zhou X, et al. Adsorption of molybdate on molybdate-imprinted chitosan/triethanolamine gel beads[J]. Carbohydrate Polymers, 2014, 114:514-520.

[5] Liu P, Jia W, Ou X, et al. Correction to Study on Synthesis and Adsorption Properties of $\mathrm{ReO}_{4}^{-}$Ion-Imprinted Polymer[J]. ACS omega, 2021, 6(14):9973.

[6] Tjasa G, Sundhirkumar S, Lea L, et al. Molecularly imprinted polymers for the removal of antide-pressants from contaminated wastewater[J]. Polymers, 2020, 13(1):120.

[7] Deng D, He Y, Li M, et al. Preparation of multi-walled carbon nanotubes based magnetic multi-template molecularly imprinted polymer for the adsorption of phthalate esters in water samples[J]. Environmental Science and Pollution Research, 2020, 28(5):5966-5977.

[8] Zhao L, Chai M, Yao H, et al. Molecularly imprinted polymers doped with carbon nanotube with aid of metal-organic gel for drug delivery systems[J]. Pharmaceutical research, 2020, 37(10):193.

[9] Zhang X, Wang H, Sun X. Preparation and properties of thermo-sensitive surface $\mathrm{Pb}(\mathrm{II})$ ion-imprinted polymers[J]. Colloids and Surfaces A, 2019, 577:138-146.

[10] He J, Shang H, Zhang X, et al. Synthesis and application of ion imprinting polymer coated magnetic multi-walled carbon nanotubes for selective adsorption of nickel ion[J]. Applied Surface Science, 2018, 428:110-117.

[11] Taghizadeh M, Hassanpour S. Selective adsorption of $\mathrm{Cr}(\mathrm{VI})$ ions from aqueous solutions using a $\mathrm{Cr}(\mathrm{VI})$-imprinted polymer supported by magnetic multiwall carbon nanotubes[J]. Polymer, 2017, 132:1-11.

[12] Huang Y, Wang R. An efficient lithium ion imprinted adsorbent using multi-wall carbon nanotubes as support to recover lithium from water[J]. Journal of Cleaner Production, 2018, 205:201-209.

[13] Santos W, Santhiago M, Yoshida I, et al. Electrochemical sensor based on imprinted sol-gel and nanomaterial for determination of caffeine[J]. Sensors \& Actuators B Chemical, 2012, 166(6):739-745.

[14] Fallah N, Taghizadeh M, Hassanpour S. Selective adsorption of Mo(VI) ions from aqueous solution using a surfacegrafted Mo(VI) ion imprinted polymer[J]. Polymer, 2018, 144:80-91.

[15] He H, Gan Q, Feng C. An Ion-imprinted Silica Gel Polymer Prepared by Surface Imprinting Technique Combined with Aqueous Solution Polymerization for Selective Adsorption of Ni(II) from Aqueous Solution[J]. Chinese Journal of Polymer Science,2018,36(04):462-471.

[16] Wei P, Li Z, Zhao X, et al. $\mathrm{Fe}_{3} \mathrm{O}_{4} / \mathrm{SiO}_{2} / \mathrm{CS}$ surface ion-imprinted polymer modified glassy carbon electrode for highly sensitivity and selectivity detection of toxic metal ions[J]. Journal of the Taiwan Institute of Chemical Engineers,2020, 113: 107-113.

[17] Jiang $\mathrm{H}$, Zhang $\mathrm{Y}$, Chen R, et al. Preparation of ion imprinted magnetic $\mathrm{Fe}_{3} \mathrm{O}_{4}$ nanoparticles for selective remediation of $\mathrm{Pb}$ (II)[J]. Journal of the Taiwan Institute of Chemical Engineers, 2017, 80:184-191.

[18] Alizadeh T, Sabzi R, Alizadeh H. Synthesis of nano-sized cyanide ion-imprinted polymer via non-covalent approach and its use for the fabrication of a $\mathrm{CN}^{-}$-selective carbon nanotube impregnated carbon paste electrode[J]. Talanta, 2016, 147:90-97.

[19] Zhang M, Zhang Z, Liu Y, et al. Preparation of core-shell magnetic ion-imprinted polymer for selective extraction of $\mathrm{Pb}$ (II) from environmental samples[J]. Chemical Engineering Journal, 2011, 178:443-450.

[20] Shao H, Wang J, Wang Q, et al. Synthesis and Application of Zwitterionic Magnetic Molecularly Imprinted Polymer for Selective Removal of Fluoroquinolones from Aqueous Solution[J]. Current Analytical Chemistry, 2021, 17(3).

[21] Zhu C, Hu T, Tang L, et al. Highly efficient extraction of lead ions from smelting wastewater, slag and contaminated soil by two-dimensional montmorillonite-based surface ion imprinted polymer absorbent[J]. Chemosphere, 2018, 209:246- 
255.

[22] Rahangdale D, Kumar A. Chitosan as a substrate for simultaneous surface imprinting of salicylic acid and cadmium[J]. Carbohydrate Polymers, 2018, 202:334-344.

[23] Yang W, Jiao F, Zhou L, et al. Molecularly imprinted polymers coated on multi-walled carbon nanotubes through a simple indirect method for the determination of 2,4-dichlorophenoxyacetic acid in environmental water[J]. Applied Surface Science, 2013, 284(11):692-699.

[24] Qin L, Zhao Y, Wang L, et al. Preparation of ion-imprinted montmorillonite nanosheets/chitosan gel beads for selective recovery of $\mathrm{Cu}$ (II) from wastewater[J]. Chemosphere,2020,252:126560.

[25] Wang A, Sun X, Li B, et al. Preparation of carbon-iron composites materials and studies of its adsorption properties for the methylene blue[J]. Journal of Inorganic and Organometallic Polymers and Materials, 2021, (31):1293-1303.

[26] Cha J, Kang S, Ali I, et al. Humic acid enhances heat stress tolerance via transcriptional activation of Heat-Shock Proteins in Arabidopsis[J]. Scientific Reports, 2020, 10(1):810-832. 
Figures

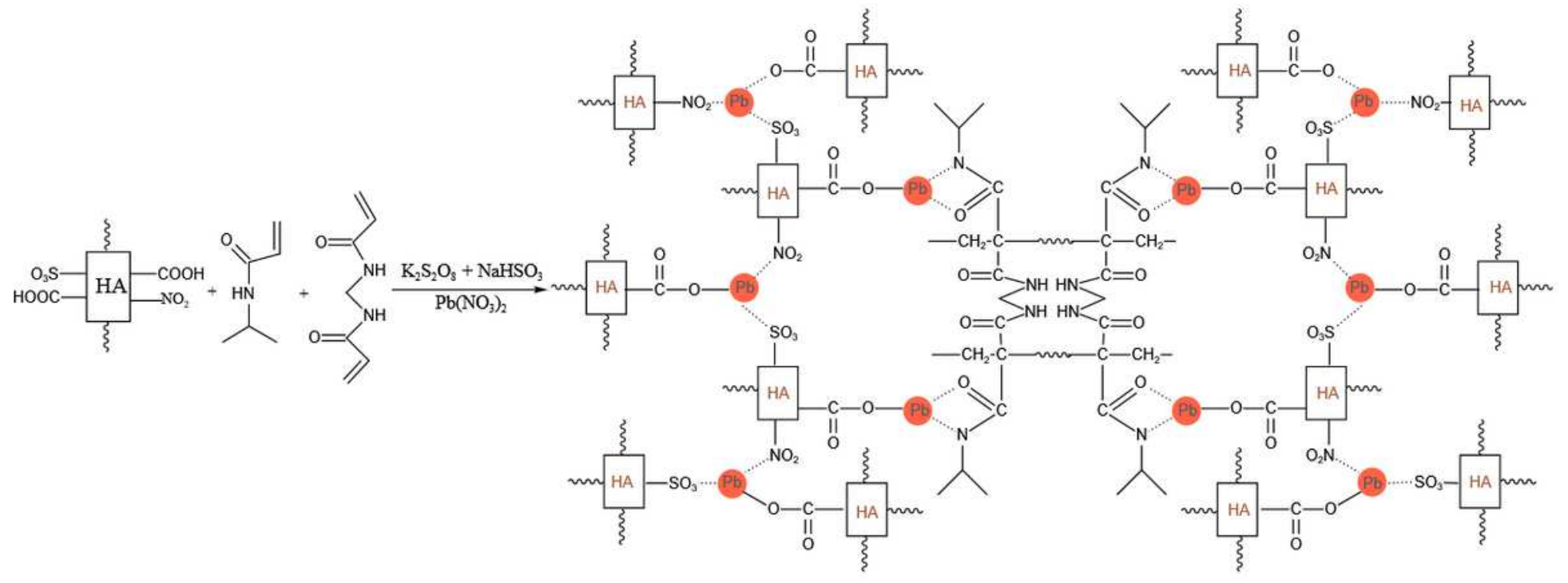

Figure 1

Preparation process of imprinted polymer.

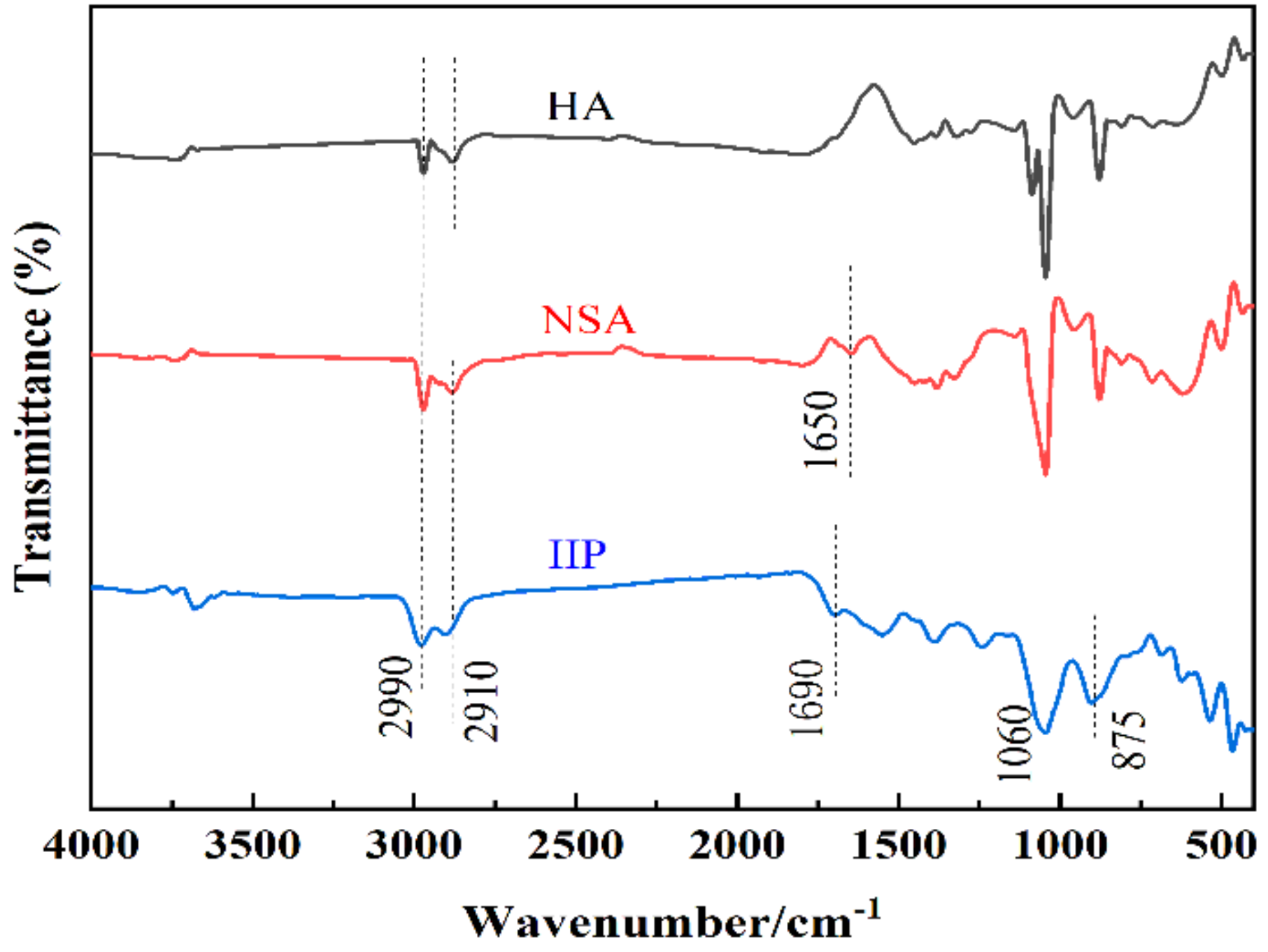


Figure 2

Infrared spectra of HA, NSA, IIPs (NSA/NIPAM).

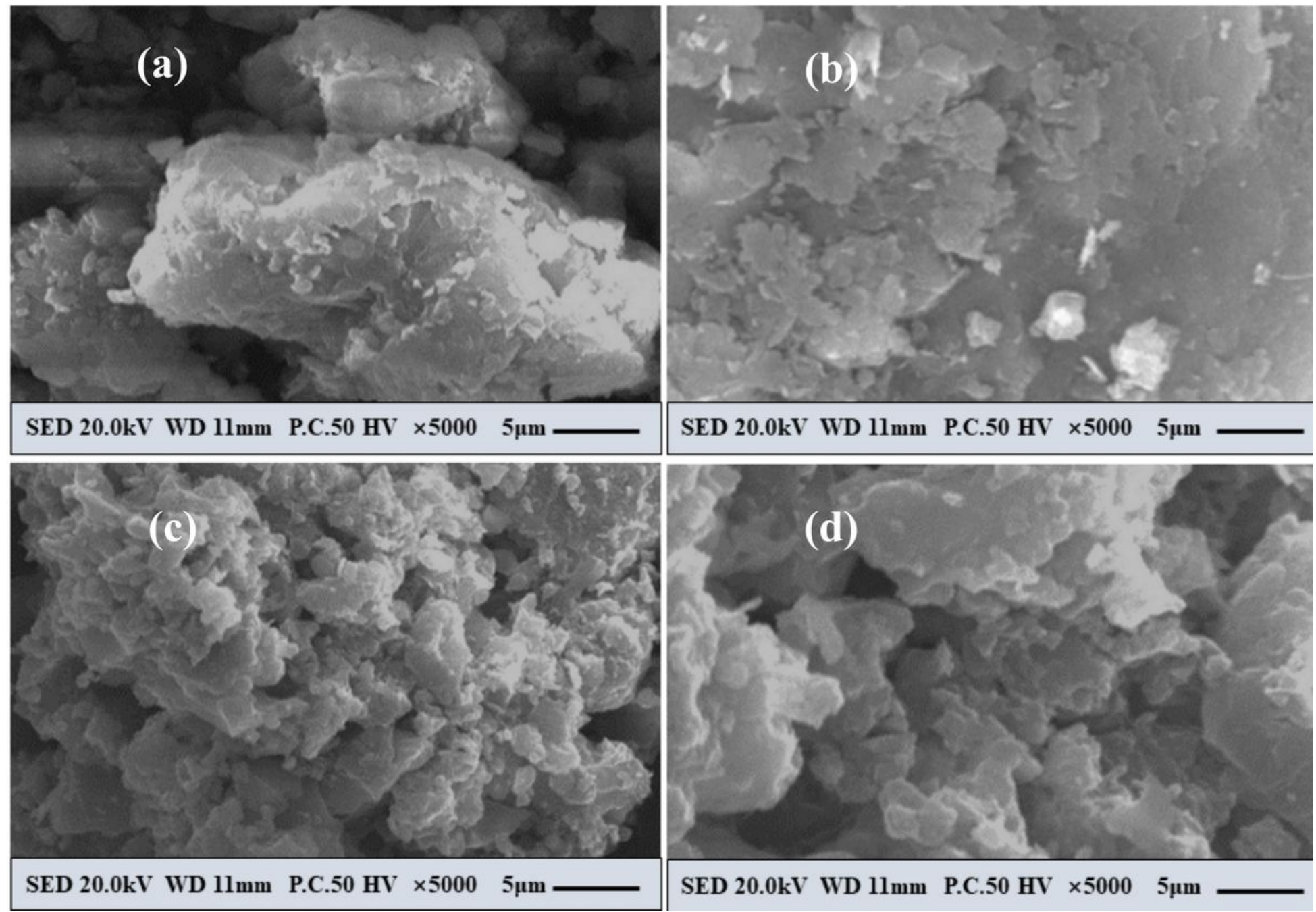

Figure 3

NSA×5000 (a); NSA×10000 (b); IIPs(NSA/NIPAM)×5000 (c) and Pb2+-IIPs(NSA/NIPAM)×10000 (d) Scanning electron micrograph 

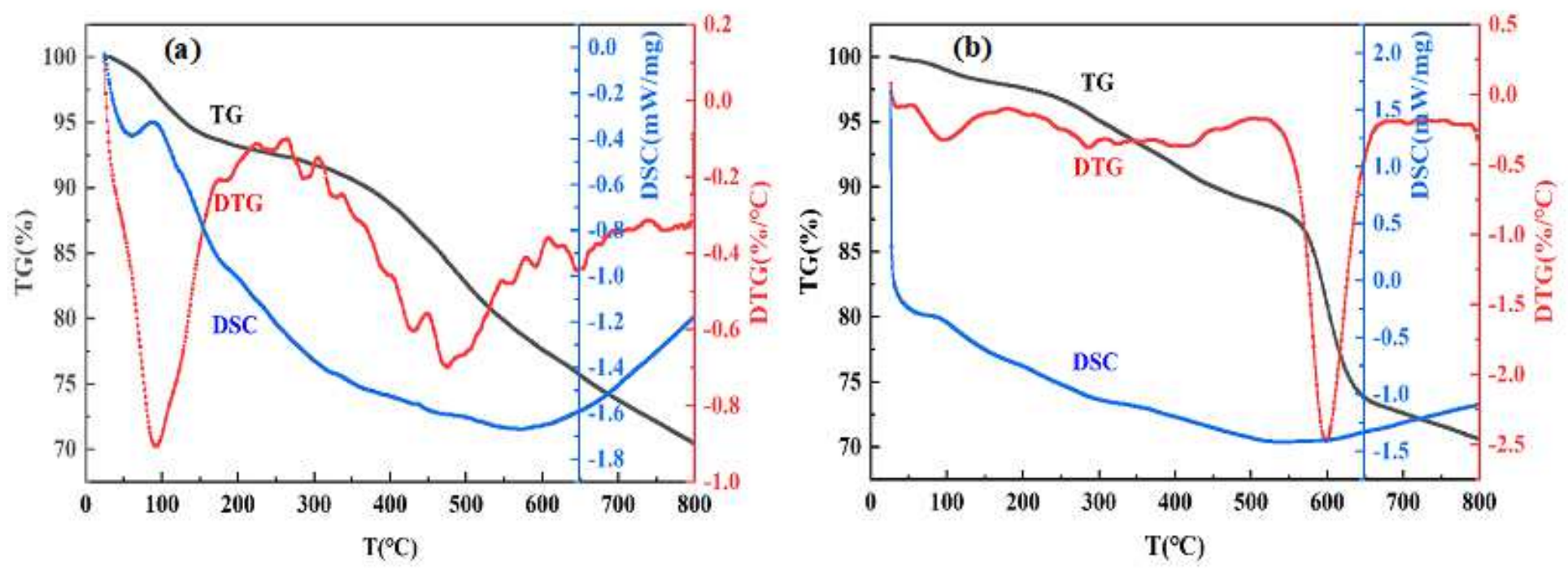

Figure 4

NSA (a) and IIPs(NSA/NIPAM ) (b) TG-DTG-DSC thermogravimetric analysis chart
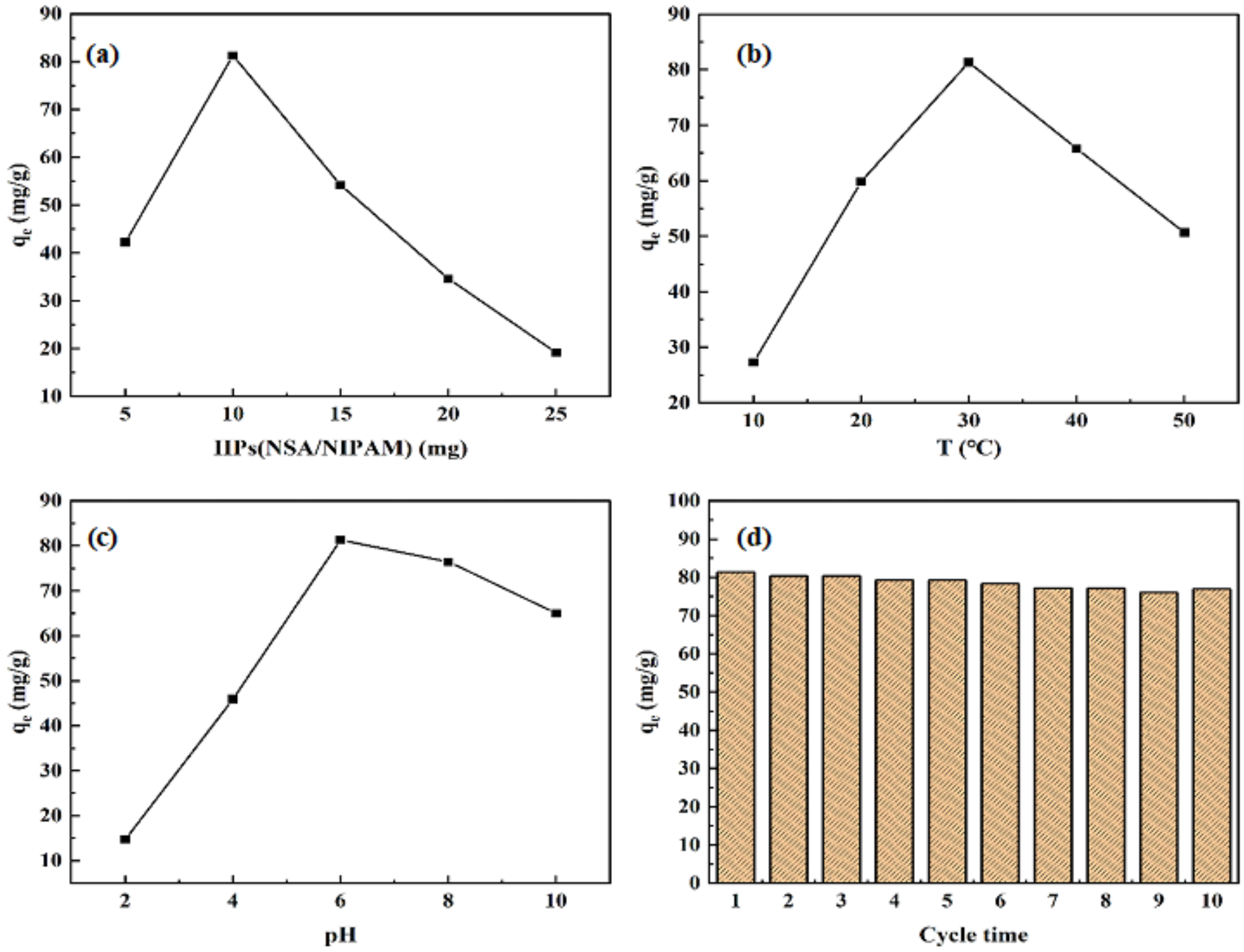
Figure 5

The effect of the amount of IIPs (NSA/NIPAM) on the adsorption capacity (a); The effect of temperature on adsorption capacity (b); The effect of pH on adsorption capacity (c); Cyclic adsorption capacity (d)

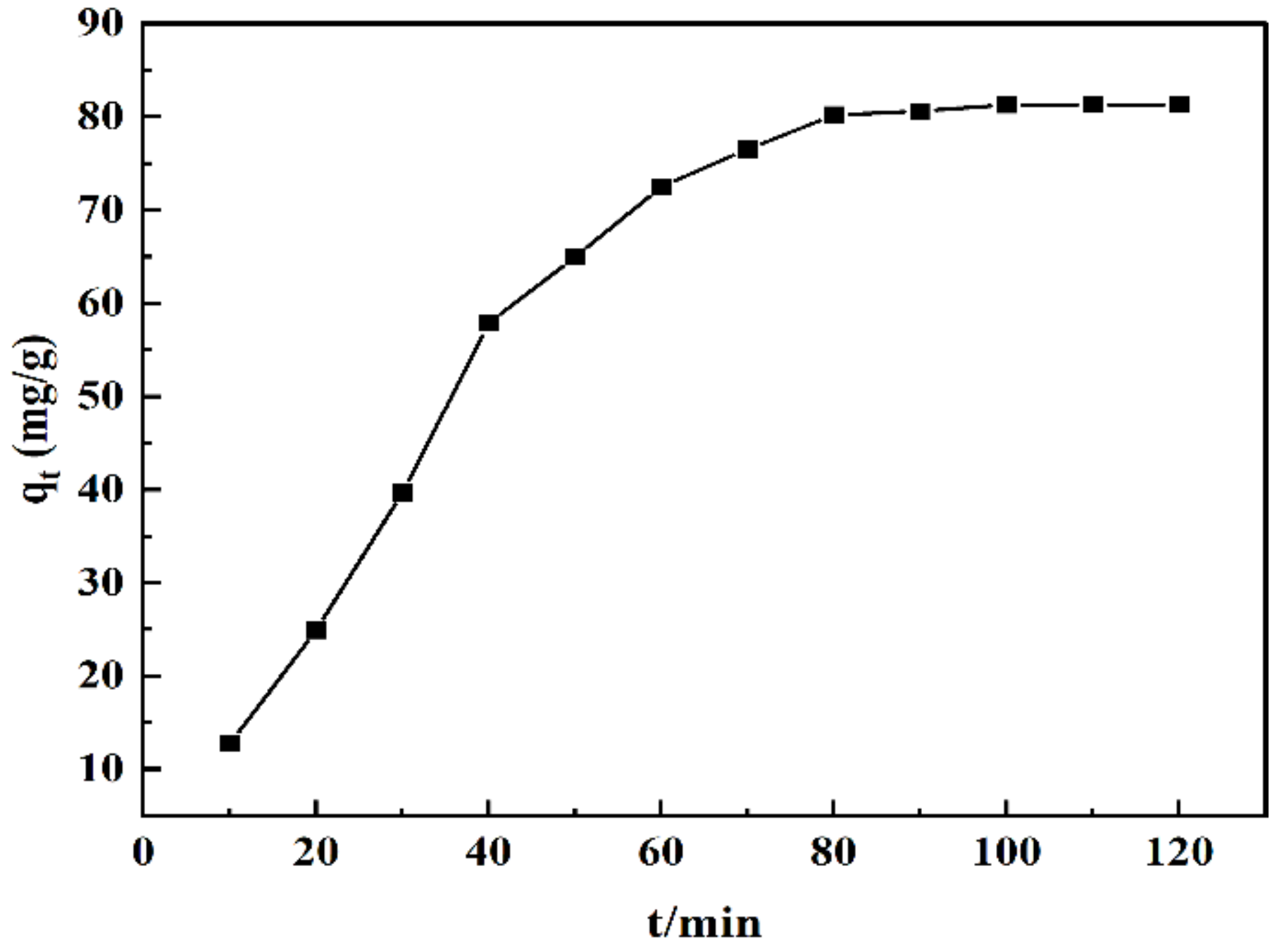

Figure 6

Change of adsorption capacity with time 

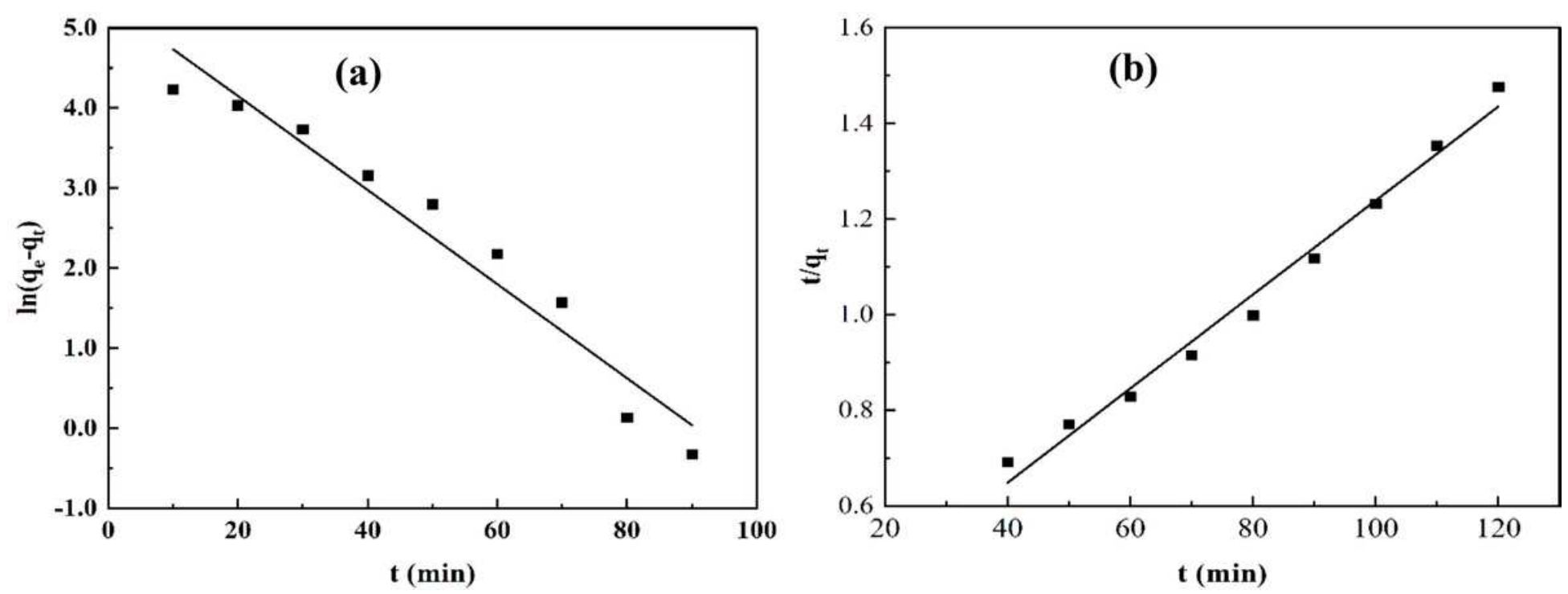

Figure 7

Kinetics of adsorption of Pb2+ by IIPs (NSA/NIPAM) Quasi-first order kinetics fitting curve (a) and Quasisecond order kinetics fitting curve (b).
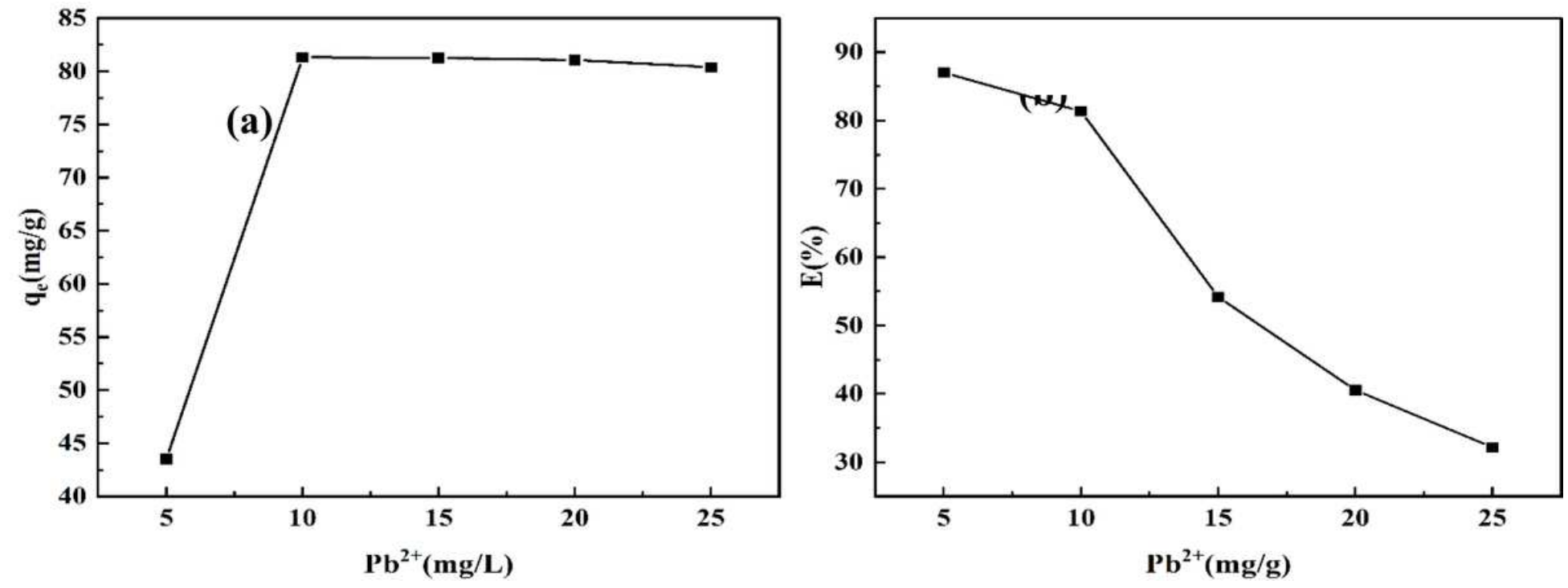

Figure 8

The influence of the initial concentration of $\mathrm{Pb} 2+$ solution on the adsorption capacity (a) and removal rate (b). 

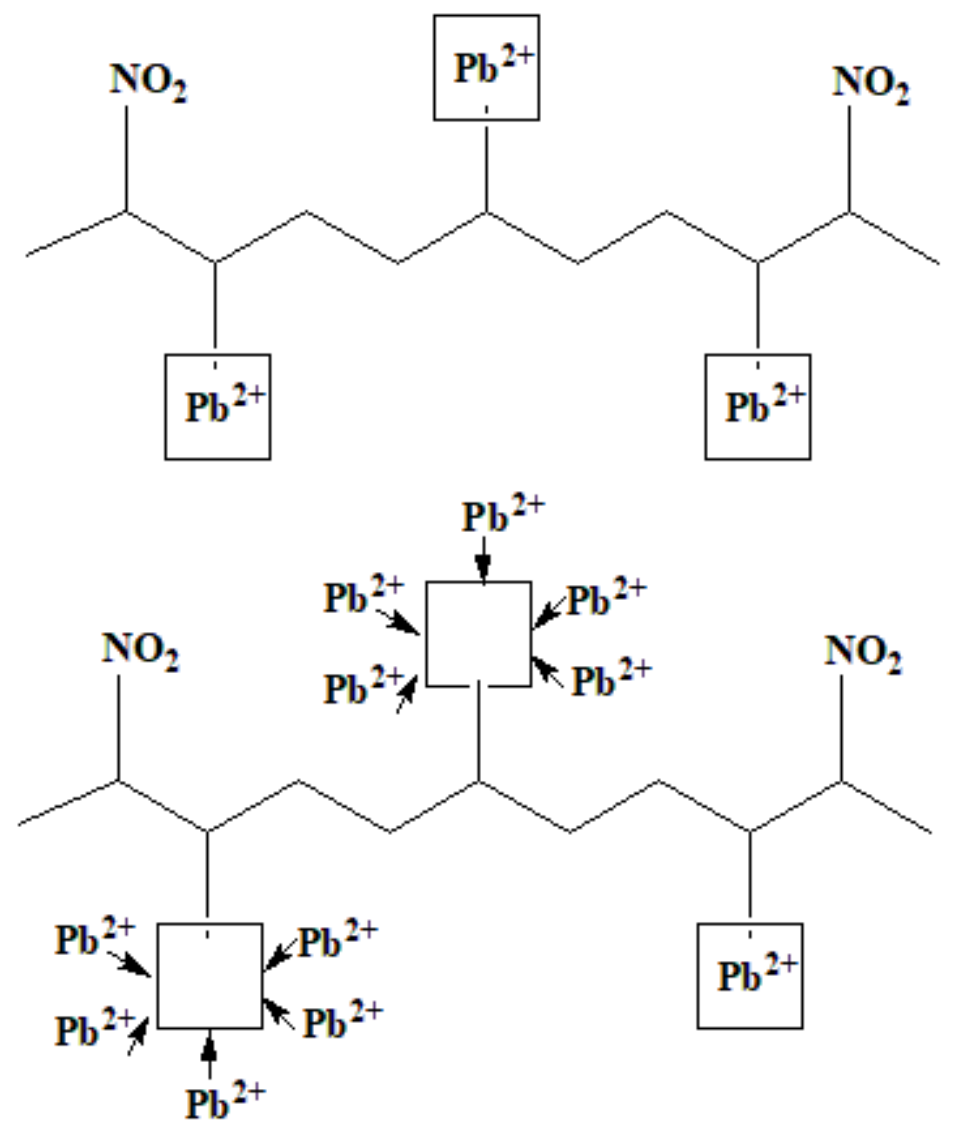

Figure 9

Schematic diagram of adsorption mechanism.
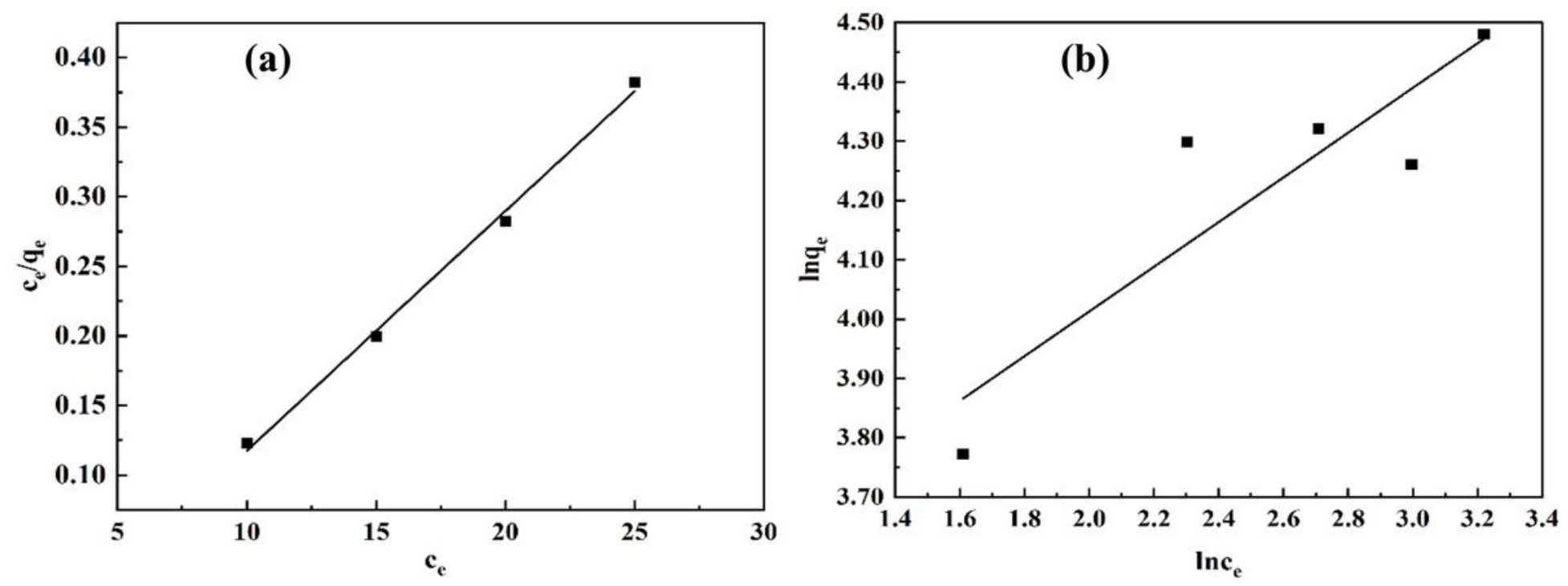

Figure 10

The Langmuir(c) and Freundlich(d) adsorption isotherm model. 